\title{
Pulmonary Pressure Necrosis due to Chronic Pleural Effusion after Heart Transplantation: A Case Report
}

\author{
Jae Seung Jung ${ }^{1}$ Sung Ho Lee ${ }^{1}$ Jeong Hyeon Lee ${ }^{2}$ Kyung Sun ${ }^{1}$ \\ ${ }^{1}$ Department of Thoracic and Cardiovascular Surgery, Korea \\ University Medical Center, Seoul, Republic of Korea \\ 2 Department of Pathology, Korea University Medical Center, Seoul, \\ Republic of Korea

\begin{abstract}
Address for correspondence Jae Seung Jung, MD, PhD, Department of Thoracic and Cardiovascular surgery, Korea University Medical Center, 73, Inchon-ro, Seongbuk-gu, Seoul 136705, Republic of Korea (e-mail: heartistcs@korea.ac.kr; heartistcs@naver.com).
\end{abstract}

Thorac Cardiovasc Surg Rep 2014;3:45-47.

\section{Introduction}

Patients with congestive heart failure (CHF) generally develop pleural effusion and ascites, and a lung may collapse passively. The primary mechanism for pleural fluid formation in patients with heart failure is fluid entering the pleural space from the lung interstitium. ${ }^{1}$ Pleural fluid in a patient with $\mathrm{CHF}$ is most likely the result of cardiac cirrhosis with ascites and transdiaphragmatic movement into the pleural space or possibly paradoxical motion of the intraventricular septum causing left ventricular diastolic dysfunction. ${ }^{2}$ Diuretics (particularly loop diuretics) remain the most effective medication for relieving symptoms and improving the pathophysiological status of fluid overload, including heart failure. ${ }^{3}$ However, if symptoms (dyspnea, orthopnea, etc.) are not relieved despite using appropriate diuretics, then the thoracentesis or paracentesis should be considered. Although both therapeutic methods can improve symptoms rapidly, repeated procedures may cause side effects such as infection, fibrin formation, and so on. Therefore, avoiding repeated attempts is better if the patient remains tolerable. ${ }^{4}$ Usually, these conditions resolve after heart transplantation (HT) and a collapsed lung will expand to near-normal size after clearance of the effusion and ascites. However, our patient's RLL of the lung remained collapsed after HT.

\section{Case Report}

A 51-year-old woman had severe restrictive cardiomyopathy, and her first symptoms started 12 years ago. Despite appropriate medical therapy, her symptoms gradually worsened. She was treated with diuretics, intermittent thoracentesis, and paracentesis due to recurrent pleural effusion and ascites. After the thoracentesis, the right lower lobe (RLL) of the lung expanded; however, it collapsed repeatedly during the follow-up period (-Fig. 1A). We stopped thoracentesis and paracentesis and added continuous intravenous diuretics for 3 months before HT because of the possibility of infection and received

February 17, 2014 accepted after revision

March 10, 2014

published online

May 29, 2014
DOI http://dx.doi.org/

10.1055/s-0034-1376178. ISSN 2194-7635. (c) 2014 Georg Thieme Verlag KG
Stuttgart . New York

License terms

(요 (1) $\Theta$ 


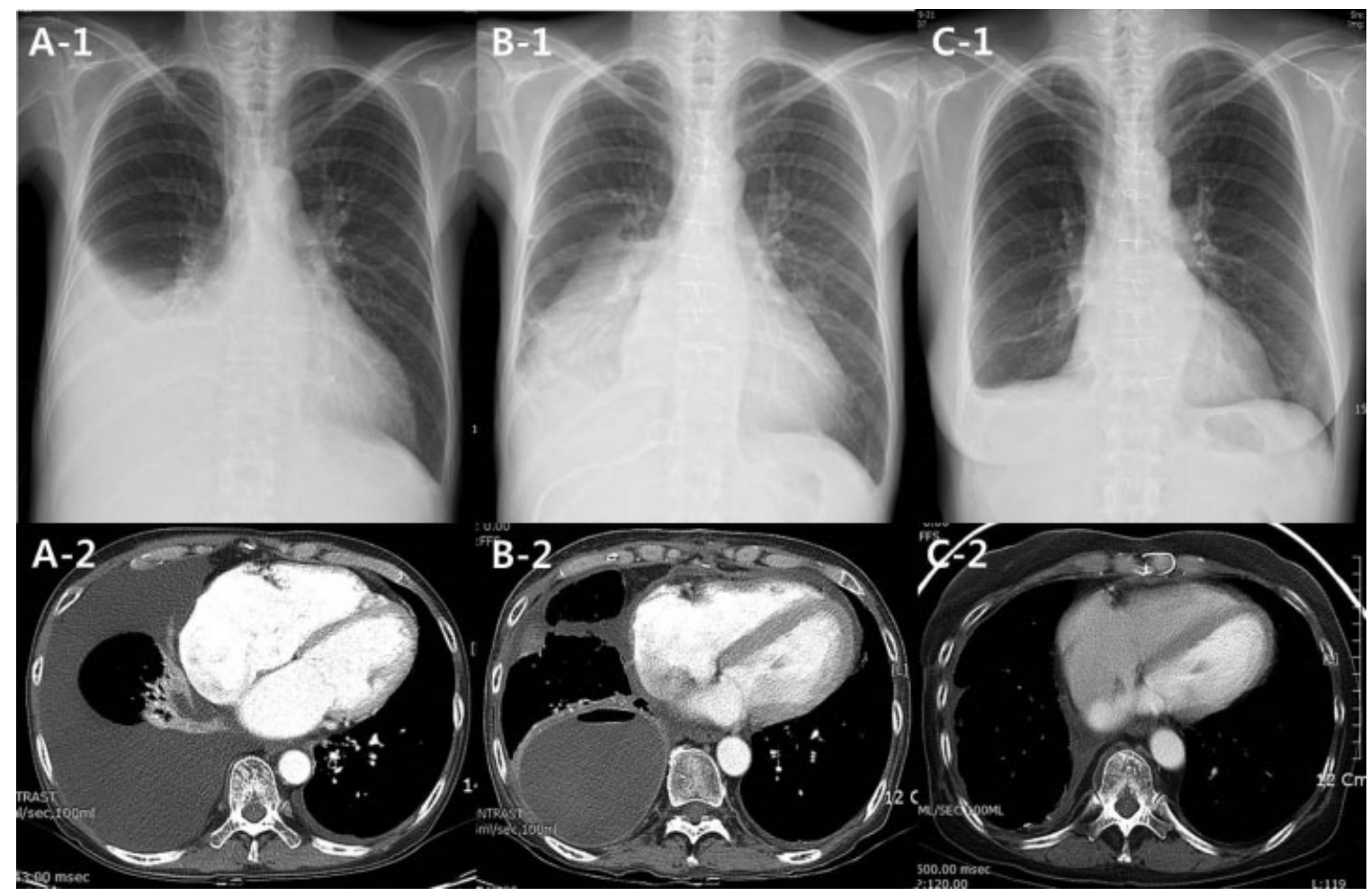

Fig. 1 Chest X-ray and computed tomography scan. (A) Before heart transplantation. Both images show right pleural effusion and collapsed right lower lobe (RLL) of a lung. (B) The RLL remains collapsed after heart transplantation (B-1). A large cystic lesion was observed in the in RLL (B-2). (C) All lesions were resolved with minimal pleural effusion after the RLL lobectomy.

trauma caused by the repetitive procedure. She finally underwent HT, but her RLL remained collapsed, and chest tube drainage persisted. We failed to find the cause of restrictive cardiomyopathy, a pathology finding showed just an intersti- tial fibrosis, and the Congo red stain was negative for amyloidosis. After rechecking the computed tomographic (CT) scan (-Fig. 1B), we performed video-assisted thoracic surgery (VATS)-assisted RLL lobectomy through a mini-

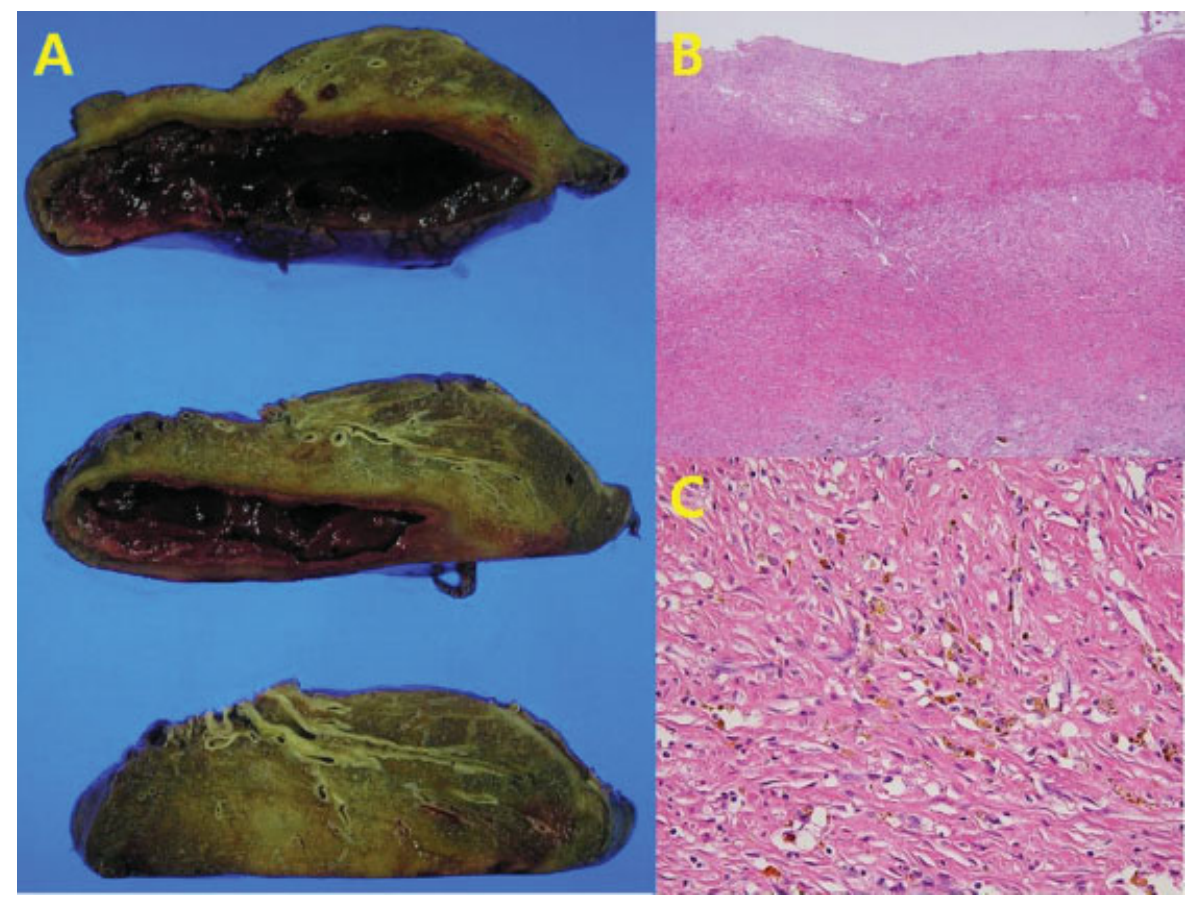

Fig. 2 (A) Gross examination shows a relatively demarcated cystic lesion filled with semisolid hemorrhagic material. This cystic lesion was confined within lung parenchyma, and did not involve the bronchial system. (B) The cystic wall shows a fibrotic capsule overlying fibrinoid material without any epithelial lining (hematoxylin and eosin $[\mathrm{H \& E}], \times 40$ ). (C) The fibrotic capsule consisted of fibroblasts intermingled with hemosiderinladen macrophages, suggestive of an old hemorrhage $(\mathrm{H} \& \mathrm{E}, \times 200)$. 
posterolateral thoracotomy (15-cm incision). Intraoperative findings revealed no effusion and the lung could not be deflated due to the cystic cavity (-Fig. 2A). No specific pathological findings were revealed except RLL necrotic lesions. A large cavitary lesion with a thick capsule was found ( - Fig. 2A, B). The capsule of the cavity showed diffuse fibrosis with intermingled hemosiderin-laden macrophages. The lumen of the cavitary lesion was filled with inflammatory cells, predominantly neutrophils, in the background of fibrinoid material (-Fig. 2C). She had not shown any complications (-Fig. 1C). She has no dyspnea, infection, or exercise limitations for the past 4 years.

\section{Discussion}

Diuretic therapy is recommended to restore and maintain normal volume status in patients with clinical evidence of fluid overload, generally manifested by congestive symptoms (orthopnea, edema, and shortness of breath), or signs of elevated filling pressure (jugular venous distention, peripheral edema, pulsatile hepatomegaly, and rales). Loop diuretics rather than thiazide-type diuretics are typically necessary to restore normal volume status in patients with $\mathrm{HF}^{5}$ However, if symptoms are not relieved despite appropriate diuretics, thoracentesis or paracentesis should be considered. Although thoracentesis can improve symptoms rapidly, repeated procedures may cause side effects such as infection, fibrin formation, pneumothorax, and so on. Chung et al reported that repeated thoracentesis increases vascular endothelial growth factor, proinflammatory cytokines (interleukin $1 \beta$, tumor necrosis factor- $\alpha$ ) and plasminogen activator inhibitors in pleural fluid. These increase fibrin formation and interrupt reabsorption of effusion, which may result in fibrin deposition and impaired resolution of pleural transudates. ${ }^{4}$ The patient received a multiple series of thoracentesis, but pleural effusion recurred repeatedly. Therefore, we stopped the procedure and infused intravenous dobutamine and loop diuretics on admission. She received a new heart after 3 months. But against our expectations, the RLL of the lung did not expand and pleural drainage $>150 \mathrm{~mL} /$ day was continuously produced. We found cystic lesions in the RLL on follow-up CT scan (-Fig. 1C) and performed a VATSassisted RLL lobectomy.

The common causes of pulmonary necrosis are bacterial pneumonia and pulmonary tuberculosis, and they both cause vascular and bronchial obstructions. No signs of vascular or bronchial obstructions were observed in our case.

In this case, the pulmonary necrosis was caused by mechanical compression without bronchial or vascular obstruction and infection. We may consider pleural effusion more actively (preoperative bronchoscopy or opening the pleura to check effusion status during HT) if we encounter a similar patient in the future. We report a rare case of pulmonary pressure necrosis on the RLL of the lung caused by mechanical compression due to chronic pleural effusion after HT.

\section{References}

1 Kinasewitz GT, Jones KR. Effusions from cardiac diseases. In: Light RW, Gary Lee YC, eds. Textbook of Pleural Diseases. 2nd ed. London: Hodder Arnold; 2008:315-321

2 Porcel JM. Pleural effusions from congestive heart failure. Semin Respir Crit Care Med 2010;31(6):689-697

3 Bellomo R, Prowle JR, Echeverri JE. Diuretic therapy in fluidoverloaded and heart failure patients. Contrib Nephrol 2010; 164:153-163

4 Chung CL, Yeh CY, Sheu JR, Chen YC, Chang SC. Repeated thoracenteses affect proinflammatory cytokines, vascular endothelial growth factor, and fibrinolytic activity in pleural transudates. Am J Med Sci 2007;334(6):452-457

5 Heart Failure Society of America. Executive Summary: HFSA 2006 Comprehensive heart failure practice guideline. J Card Fail 2006; $12: 10-38$ 